Stochastics and Dynamics

(C) World Scientific Publishing Company

\title{
ROBUST ASYMPTOTIC CONTROLLABILITY UNDER TIME-VARYING PERTURBATIONS
}

\author{
LARS GRÜNE \\ Mathematisches Institut, Universität Bayreuth, 95440 Bayreuth, Germany \\ lars.gruene@uni-bayreuth.de
}

Received February 27, 2004

\begin{abstract}
We investigate the effect of time-varying perturbations on the dynamical behavior of nonlinear control systems. More specifically, we study the effect of such perturbations on the controlled equivalent of asymptotically stable sets, i.e., asymptotically controllable sets. In the first part of this paper we illustrate by a simple example how different types of perturbations affect this dynamical behavior and use concepts from dynamical game theory in order to identify classes of perturbations which allow to model the effects of numerical discretization errors both in time and space. In the second part we introduce appropriate robustness properties and prove that these are inherent properties for asymptotically controllable sets under these classes of perturbations.
\end{abstract}

Keywords: controllability; time-varying perturbation; discretization.

AMS Subject Classification: 93B05; 93D09; 91A23; 65P40; 37B25

\section{Introduction}

The numerical investigation of the long-time behavior of dynamical systems has been an object of interest for the last two decades with a huge number of contributions centering around the questions of reliability of numerically observed dynamical behavior and on the design of efficient algorithms for the determination of such behavior ${ }^{4,6,20}$. While most of the results in this field center around autonomous dynamical systems, here we consider control systems, i.e., dynamical systems which can be influenced by a time dependent control function and which can be interpreted as a nonautonomous dynamical systems ${ }^{2}$. For such systems, the analogue of an asymptotically stable set is an asymptotically controllable set. As in the uncontrolled case such sets are characterized by the long term behavior of solutions which implies that standard finite time error estimates for numerical approximations do not allow to conclude that these sets persist under discretization. For asymptotically stable sets this persistence property was shown by arguments using the numerical scheme itself ${ }^{15}$. The approach in the present paper, however, differs from this direct line of reasoning because here we use an "embedding" of the numerical approximation into a suitably perturbed system. The main advantage of this approach is that robustness properties obtained for the perturbed system allow us to simultaneously 
conclude robustness results for large classes of different numerical discretizations of control systems, e.g., in time $\mathrm{e}^{5,9}$ or in space ${ }^{21}$.

In the context of asymptotically stable sets (or, more specifically, attractors) this perturbation technique was used by Kloeden and Kozyakin ${ }^{14}$ and in a sense our paper can be understood as a controlled equivalent to this paper. In the controlled case, however, several difficulties arise from the question of how the control function and the perturbation interact. The goal of this paper is therefore to present a framework for studying discretization effects on dynamical objects in control systems by embedding such systems in suitable perturbed systems for which asymptotic controllability can be shown to be a robust property in an appropriate sense.

More precisely, the contribution of the present paper is twofold. After fixing the setup in Section 2, the first contribution is the discussion of different possible perturbation structures in Section 3. Here we identify suitable classes of perturbations which are able to capture the effect of discretization errors. In particular, we show that a free choice of the perturbation will not allow for reasonable robustness properties (Example 3.1), we prove that certain discretizations in time can be covered by so called nonanticipative strategies (Lemma 3.1 and the subsequent discussion) and we demonstrate that this class needs to be broadened in order to cover errors from space discretizations (Discussion after Definition 3.5 and Lemma 3.2). The second contribution of this paper is found in Section 4, where it is shown that and in which sense asymptotic controllability is a robust dynamical phenomenon. The corresponding results were already formulated in Chapter 4 of Grüne ${ }^{6}$, here, however, we give alternative proofs which avoid the technical apparatus developed in this reference and close some gaps in the proofs.

\section{Setup}

In this paper we consider perturbed control systems of the form

$$
\dot{x}(t)=f(x(t), u(t), w(t))
$$

with vector field

$$
f: \mathbb{R}^{d} \times U \times W \rightarrow \mathbb{R}^{d}
$$

being continuous and globally Lipschitz in $x$ uniformly for all $u$ and $w$, control functions

$$
u \in \mathcal{U}:=\{u: \mathbb{R} \rightarrow U \mid u \text { measurable }\}
$$

and time-varying perturbations

$$
w \in \mathcal{W}:=\{w: \mathbb{R} \rightarrow W \mid w \text { measurable }\},
$$

where $U \subset \mathbb{R}^{m}$ and $W \subset \mathbb{R}^{l}$ are the control and perturbation ranges which we consider as compact. The solutions of (2.1) for initial value $x$ at initial time $t=0$ will be denoted by $\varphi(t, x, u, w)$. Note that our assumptions can be weakened in 
many ways. In particular, the compactness and global Lipschitz assumptions are only imposed in order to simplify the presentation of the proofs and can be relaxed by suitable cutoff techniques since we are interested in the behavior of the system only on compact subsets of the state space.

We want to study the influence of the time--varying perturbations $w(t)$ on asymptotically controllable sets $A$. More precisely, using the notion of $\mathcal{K} \mathcal{L}$ functions ${ }^{\mathrm{a}}$ and denoting the Euclidean distance to a set $A$ by $\|\cdot\|_{A}$ we consider the following objects.

Definition 2.1. A compact set $A \subset \mathbb{R}^{d}$ is called asymptotically controllable with controllability neighborhood $B$ of $A$, if there exists a function $\beta \in \mathcal{K} \mathcal{L}$ such that for each $x \in B$ there exists $u_{x} \in \mathcal{U}$ with

$$
\left\|\varphi\left(t, x, u_{x}, 0\right)\right\|_{A} \leq \beta\left(\|x\|_{A}, t\right)
$$

for all $t \geq 0$.

This definition generalizes the concept of asymptotic stability to control systems and is standard in mathematical control theory, cf. e.g. Sontag ${ }^{18}$. Several other stability concepts for control systems were investigated by Kloeden ${ }^{13}$, in particular the eventual uniform asymptotic weak stability in this reference is closely related to our notion.

The aim of this paper is to analyze what happens to this asymptotic controllability property if we "turn on" the perturbation $w$, i.e., consider the trajectories $\varphi(t, x, u, w)$ instead of $\varphi(t, x, u, 0)$. Our goal is to derive a robustness statement for the sets $A$ guaranteeing that approximate controllability is preserved under perturbations, where "approximate" will be made precise later.

The behavior of the perturbed system crucially depends on what kind of perturbations we allow, i.e., we will need to pick the right perturbation classes in order to be able to prove robustness properties. On the other hand, the choice of perturbation classes depends on modeling issues, i.e., which kind of perturbation influence we want to be present in our system. Here we will address discretization phenomena by considering perturbations that are induced by the effect of discretization errors.

\section{Discretization and perturbation}

In this section we want to study the effects of discretizations in both time and space and identify suitable classes of perturbations for modeling these effects. We consider

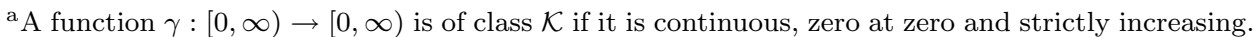
A function $\beta:[0, \infty) \times[0, \infty) \rightarrow[0, \infty)$ is of class $\mathcal{K} \mathcal{L}$ if it is continuous, of class $\mathcal{K}$ in the first variable and strictly decreasing to 0 in the second variable. The use of $\mathcal{K}$ and $\mathcal{K} \mathcal{L}$ functions in stability theory for ODEs apparently goes back to Hahn's books ${ }^{10,11}$, where these classes are introduced without further explanation of the nomenclature. Conceptually similar functions have already been used earlier by Müller ${ }^{16}$ and Kamke $^{12}$ for the qualitative analysis of ODEs, which has lead to the conjecture that $\mathcal{K}$ stands for Kamke. Note, however, that in the literature one can also find a definition for Kamke functions which differs from the $\mathcal{K}$ and $\mathcal{K} \mathcal{L}$ classes ${ }^{22}$ and is in fact closer to the functions used by Kamke and Müller. 
discretizations of control systems without perturbations, i.e., systems of the form

$$
\dot{x}(t)=g(x(t), u(t))
$$

whose trajectories we denote by $\varphi(t, x, u)$ and start with the discretization in time.

\subsection{Discretization in time}

For affinely controlled nonlinear control systems, i.e., unperturbed control systems (3.2) with the special structure

$$
g(x, u)=g_{0}(x)+\sum_{i=1}^{m} u_{i} g_{i}(x)
$$

it was recently shown ${ }^{9}$ that one can derive numerical one step schemes similar to the classical Taylor- and Runge-Kutta schemes for ordinary differential equations. The main difficulty here lies in the fact that the control functions $u$ are in general allowed to be measurable in $t$ and in many applications (like, e.g., optimal control) may indeed exhibit discontinuities. Hence the classical schemes are not applicable directly, instead one needs to use modified schemes using suitable integrals over $u$. The simplest case of such a scheme is the analogue of the Euler scheme which for a control affine system and time step $h>0$ reads

$$
x_{j+1}=x_{j}+h g_{0}\left(x_{j}\right)+\sum_{i=1}^{m} \bar{u}_{i, j} g_{i}\left(x_{j}\right)
$$

with

$$
\bar{u}_{i, j}=\int_{j h}^{(j+1) h} u_{i}(t) d t .
$$

In a more abstract formulation, any such scheme can be written as

$$
x_{j+1}=\Phi_{h}\left(x_{j}, u(j h+\cdot)\right)
$$

for an appropriately chosen map $\Phi_{h}: \mathbb{R}^{d} \times \mathcal{U} \rightarrow \mathbb{R}^{d}$ involving multiple integrals over the control functions $u(j h+\cdot)$ on the interval $[0, h]$ or - equivalently - integrals over $u$ on $[j h,(j+1) h]$. By iterating these schemes from $x_{0}=x$ on can define associated discrete trajectories by

$$
\Phi_{h}(t, x, u):=x_{j} \quad \text { for } t=j h, j=0,1,2, \ldots
$$

The discretization error of (3.4) is measured by the usual consistency estimate

$$
\left\|\varphi(h, x, u)-\Phi_{h}(x, u)\right\| \leq C h^{p+1}
$$

which is supposed to hold for all sufficiently small time steps $h>0$. Here the parameter $p>0$ is called the order of the scheme and it was shown ${ }^{9}$ that in principle one can construct schemes of arbitrary order, for the Euler scheme (3.3) one obtains $p=1$. Convergence statements of these schemes are easily derived from 
(3.5) using appropriate stability (or Lipschitz) conditions which are also satisfied by the mentioned schemes ${ }^{9}$.

The goal of the construction of our perturbed system is now to define a class of perturbations such that the numerical scheme $\Phi_{h}$ can be embedded into the perturbed system (2.1) with

$$
f(x, u, w)=g(x, u)+w .
$$

This particular structure, sometimes referred to as the inflated system (see also Kloeden and Kozyakin ${ }^{14}$ for an equivalent definition via differential inclusions), allows the perturbation to change the system's trajectories in all possible directions contained in the perturbation range $W$. In order to include all possible directions we will use the particular perturbation range

$$
W_{\alpha}:=\left\{w \in \mathbb{R}^{d} \mid\|w\| \leq \alpha\right\}
$$

and denote by $\mathcal{W}_{\alpha}$ the corresponding space of perturbation functions. The desired embedding property is now given by the following definition.

Definition 3.1. We say that the numerical scheme (3.4) is embedded into the perturbed system (2.1), (3.6) for $\alpha>0$, if for any initial value $x \in \mathbb{R}^{d}$ and any control function $u \in \mathcal{U}$ there exists an admissible perturbation function $w \in \mathcal{W}_{\alpha}$ such that

$$
\varphi(t, x, u, w)=\Phi_{h}(t, x, u)
$$

holds for all $t=0, h, 2 h, \ldots$.

Since we can use the consistency estimate (3.5) it seems reasonable to expect that this embedding property can be met for perturbation range $W_{\alpha}$ with $\alpha \sim h^{p}$, and we will see later that this is indeed the case. Obviously, the discretization error will depend on the time $t$ which motivates our general setting using time-varying perturbations, because with a fixed perturbation $w \equiv$ const it will in general be impossible to satisfy (3.7). Furthermore, the perturbation $w$ will depend on $x$ and $u$ as well as on the dynamics $\varphi$ and on the choice of the numerical scheme $\Phi_{h}$. In this context the delicate question is, what kind of information about $u$ is allowed in the choice of $w$. Certainly, in order to satisfy (3.7) the perturbation needs information about the control function because the discretization error will depend on $u$. Hence, the problems we will discuss next are:

- Which information from $u$ is needed for the choice of $w$ such that (3.7) can be satisfied?

- How much information can we allow to use such that we can still obtain a robustness property for $A$ ?

A first attempt would be to choose $w$ freely from the space $\mathcal{W}_{\alpha}$ using all available information about the unperturbed system's behavior. However, the following example shows that with this choice the perturbation can completely destroy the asymptotic controllability property. 
Example 3.1. Consider the 1d control system

$$
\dot{x}(t)=u(t)+w(t) .
$$

Clearly, the unperturbed system for $w \equiv 0$ is asymptotically controllable to the set $A=\{0\}$ from $B=\mathbb{R}$, which can be seen by choosing, e.g., $u_{x}(t)=-e^{-t} x$ resulting in the solution trajectories

$$
\varphi(t, x, u, 0)=e^{-t} x
$$

which satisfy Definition 2.1 with $\beta(|x|, t)=e^{-t}|x|$. Since the perturbation can be chosen freely from $\mathcal{W}_{\alpha}$ using all available information, we can pick an arbitrary $T>0$ and set

$$
w \equiv\left\{\begin{aligned}
-\alpha, & \text { if } \varphi(T, x, u, 0) \leq 0 \\
\alpha, & \text { if } \varphi(T, x, u, 0)>0 .
\end{aligned}\right.
$$

It immediately follows that the perturbed trajectory satisfies

$$
|\varphi(T, x, u, w)| \geq T \alpha,
$$

i.e., no matter how small $\alpha>0$ is, one can always choose a perturbation which steers the system arbitrarily far away from the asymptotically controllable set $A$.

Even in this simple example a "free" choice of $w$ completely destroys the asymptotic controllability property, hence we do not obtain any reasonable "approximate" notion of controllability. The reason for this effect lies in the fact that in this setting the control was not allowed to "react" on the specific perturbation acting on the system. Thus, we need a perturbation structure which (i) allows $w$ to depend on $u$ and (ii) allows $u$ to react on the chosen perturbation $w$. Since this will in turn change $w$, again, we need a suitable information structure that allows for both (i) and (ii) without creating infinite "loops".

The solution for this problem is obtained by carefully looking at the time: in the example above in the choice of $w$ we were allowed to look into the future of the trajectories' behavior, i.e., we have used information about $\varphi(T, x, u, 0)$ at a time $T>0$ in order to design $w$ on the interval $[0, T]$. We will now introduce a class of perturbations which do not allow for using this type of information from the future. The appropriate formal concept for this has its origins in differential game theory ${ }^{23,1}$.

Definition 3.2. We define the class of nonanticipative strategies by

$$
\mathcal{P}_{\alpha}:=\left\{\begin{array}{l|l}
p: \mathcal{U} \rightarrow \mathcal{W}_{\alpha} & \begin{array}{l}
\text { for all } t \in \mathbb{R} \text { the following implication holds: } \\
u_{1}(s)=u_{2}(s) \text { for almost all } s \in(-\infty, t] \Rightarrow \\
p\left[u_{1}\right](s)=p\left[u_{2}\right](s) \text { for almost all } s \in(-\infty, t]
\end{array}
\end{array}\right\}
$$

In other words, this definition demands the following: For any perturbation $w(t)=p[u](t)$ it is still allowed to use the full information from $u$ (and the induced 
information on $\varphi$ and $\Phi_{h}$ depending on $u$ ) for the design of $w$, however, with the restriction that

- we are only allowed to use information from the past

- we are not allowed to change the past behavior of $w$ at a later time.

Once the perturbation strategy $p[u]$ is fixed, then the control $u$ may be chosen freely from $\mathcal{U}$, assuming the knowledge of $p$. This way of choosing $p$ and $u$ defines the rules of our "differential game", and we re-examine Example 3.1 for this class of perturbations in order to show that for perturbations from $\mathcal{P}$ we can obtain controllability under perturbations.

Example 3.2. We again consider the system

$$
\dot{x}(t)=u(t)+w(t)
$$

now with control range $U=[-1,1]$ and perturbation range $W=[-1 / 2,1 / 2]$. Let $p[u]$ be an arbitrary perturbation strategy. For some given time $\tau>0$ we inductively define a control function $u(t)$ by

$$
\left.u\right|_{[i \tau,(i+1) \tau]} \equiv\left\{\begin{array}{cl}
\max \{-1,-\varphi(i \tau, x, u, p[u]) / \tau\}, & \text { if } \varphi(i \tau, x, u, p[u]) \geq 0 \\
\min \{1,-\varphi(i \tau, x, u, p[u]) / \tau\}, & \text { if } \varphi(i \tau, x, u, p[u])<0 .
\end{array}\right.
$$

By induction one sees that each trajectory reaches the set $[-\tau / 2, \tau / 2]$ at a time $t \leq T=2|x|$ and stays there for all future times. Hence for the perturbed system we find asymptotically controllable sets which are arbitrarily close to $A=\{0\}$, i.e., the asymptotic controllability property is robust w.r.t. perturbations from $\mathcal{P}$.

We will show in the next section that this class indeed allows for general robustness statements of asymptotic controllability. Before we turn to this question, we need to investigate whether this class is still rich enough to achieve the embedding property from Definition 3.1. The following Lemma shows that this is the case for the Euler scheme (3.3).

Lemma 3.1. Consider the controlled Euler scheme given by

$$
\Phi_{h}(x, u)=x_{j}+h g_{0}\left(x_{j}\right)+\sum_{i=1}^{m} \int_{0}^{h} u_{i}(t) d t g_{i}\left(x_{j}\right)
$$

for some step size $h>0$. Then for each $x \in \mathbb{R}^{d}$ there exists a nonanticipative strategy $p \in \mathcal{W}_{\alpha}$ for $\alpha=K h$ and some suitable $K>0$, such that (3.7) is satisfied with $w(t)=p[u](t)$.

Proof. We first show (3.7) for $t=h$. In order to prove this observe that the map

$$
t \mapsto \Phi_{t}(x, u)
$$

is differentiable almost everywhere and satisfies

$$
\frac{d}{d t} \Phi_{t}(x, u)=g_{0}(x)+\sum_{i=1}^{m} u_{i}(t) g_{i}(x)=g(x, u(t))=f(x, u(t), 0)
$$


for almost all $t \in[0, h]$. Now we define the perturbation strategy $p[u]$ for $t \in[0, h]$ as

$$
p[u](t)=f(x, u(t), 0)-f\left(\Phi_{t}(x, u), u(t)\right) .
$$

This strategy satisfies Definition 3.2 because if $u_{1}$ and $u_{2}$ coincide for almost all $s \in[0, t]$, then also the quantities defining $p\left[u_{1}\right]$ and $p\left[u_{2}\right]$ coincide, hence we obtain $p\left[u_{1}\right](s)=p\left[u_{2}\right](s)$ for almost all $s \in[0, t]$.

In order to see (3.7), observe that on the one hand we have

$$
\frac{d}{d t} \varphi(t, x, u, p[u])=f(\varphi(t, x, u, p[u]), u(t), 0)+p[u](t)
$$

and on the other hand one computes

$$
\frac{d}{d t} \Phi_{t}(x, u)=f(x, u(t), 0)=f\left(\Phi_{t}(x, u), u(t), 0\right)+p[u](t) .
$$

Thus, $\varphi(t, x, u, p[u])$ and $\Phi_{t}(x, u)$ satisfy the same Carathèodory differential equation and since their initial value $x$ at $t=0$ coincides, by uniqueness of solutions we can conclude (3.7).

Finally, we show that $p \in \mathcal{P}_{\alpha}$, i.e., $p[u](t) \in W_{\alpha}$ with $\alpha=K h$ for some suitable $K>0$ almost all $t \in[0, h]$. This follows immediately from

$$
\|p[u](t)\|=\left\|f(x, u(t), 0)-f\left(\Phi_{t}(x, u), u(t), 0\right)\right\| \leq L\left\|\Phi_{t}(x, u)-x\right\| \leq L M t \leq K h
$$

with $M:=\max _{u \in U}\|f(x, u)\|$ and $K=L M$.

For the times $t=j h$ with $j \geq 2$ the proof is obtained by induction: on each interval $I_{j}=[j h,(j+1) h]$ we repeat the construction from above replacing $x$ by $x_{j}=\Phi(j h, x, u)$, which defines $p[u]$ on $I_{j}$. Since this choice of $p[u]$ only depends on the past values of $u$ we indeed obtain a nonanticipative strategy.

A similar construction can be applied to the higher order schemes developed by Grüne and Kloeden ${ }^{9}$, where, however, an appropriate Taylor series expansion of $\varphi(t, x, u)$ is needed, see Proposition 5.2.14 in Grüne ${ }^{6}$. Indeed, it seems plausible to conjecture that any reasonable discretization in time can be embedded using perturbations generated by nonanticipative strategies $\mathcal{P}$. In the next section we show that this is no longer the case when we consider spatial discretizations.

\subsection{Discretization in space}

In many algorithms for the actual computation of dynamical objects like attractors, invariant manifolds or - in our setting - asymptotically controllable sets, a discretization of the underlying state space is used in order to represent the desired objects on a finite "basis" of sets. The method we describe next is based on a cell discretization of the space and was motivated by the subdivision algorithm developed by Dellnitz and Hohmann ${ }^{3}$, but similar arguments are valid for other types of discretizations, like, e.g., quantization techniques which are often used in control theory. 
Formally, our cell discretization can be described as follows.

Definition 3.3. Consider a compact set $\Omega \subset \mathbb{R}^{d}$.

(i) A cell covering $\mathcal{Q}=\left(Q_{i}\right)_{i=1, \ldots, P}$ of $\Omega$ is a finite family of closed sets $Q_{i}$, $i=1, \ldots, P, P \in \mathbb{N}$, with $Q_{i}=\overline{\operatorname{int} Q_{i}}$, such that $\operatorname{int} Q_{i} \cap \operatorname{int} Q_{j}=\emptyset$ for all $i \neq j$ and $\bigcup_{i=1, \ldots, P} Q_{i}=\Omega$ holds. The sets $Q_{i}$ are called the cells of the covering.

(ii) With $\mathcal{C}_{\mathcal{Q}}$ we denote the set of all possible unions of cells in $\mathcal{Q}$, i.e.,

$$
\mathcal{C}_{\mathcal{Q}}:=\left\{C \subseteq \Omega \mid C=\bigcup_{i \in I} Q_{i} \text { for an arbitrary index set } I \subseteq\{1, \ldots, P\}\right\} .
$$

(iii) The value $\operatorname{diam}\left(Q_{i}\right):=\max _{x, y \in Q_{i}}\|x-y\|$ is called the diameter of the cell $Q_{i}$ and the value $\operatorname{diam}(\mathcal{Q}):=\max _{i=1, \ldots, P} \operatorname{diam}\left(Q_{i}\right)$ is called the diameter of the covering $\mathcal{Q}$.

Next we define an approximation of our control system $(3.2)$ on $\mathcal{C}_{\mathcal{Q}}$.

Definition 3.4. For a given time step $h>0$ a space discretization of (3.2) is a set valued map $\Psi: \Omega \times \mathcal{U} \rightarrow \mathcal{C}_{\mathcal{Q}}$ satisfying

$$
\Psi(x, u)=Q_{i},
$$

for some cell $Q_{i} \in \mathcal{Q}$ with $\varphi(h, x, u) \in Q_{i}$ and

$$
\Psi(x, u)=\emptyset
$$

if $\varphi(h, x, u) \notin \Omega$.

Note that the above definition does not uniquely determine the set valued map $\Psi$, it just requests that $\Psi$ assigns to each pair $(x, u)$ a cell $Q_{i}$ with $\varphi(h, x, u) \in Q_{i}$. We emphasize that this definition describes a theoretical discretization which cannot in general be implemented numerically. For implementational issues we refer to Chapter 5 of Grüne ${ }^{6}$. Observe that the resulting value only depends on the piece $\left.u\right|_{[0, h]}$ of the chosen control function.

In order to iterate the map $\Psi$, we need to pick a point $x_{1} \in \Psi(x, u)$ to which we can apply $\Psi$ again using the next piece $\left.u\right|_{[h, 2 h]}$ of the control function. However, taking into account that we want to model the influence of "bad" perturbations we are not free to choose this point $x_{1}$. Instead, we model the fact that there is a perturbation acting on the system which determines the point $x_{1} \in \Psi(x, u)$ to be chosen for the next step. Once $x_{1}$ is determined we can apply the map $\Psi$ once again and define its second iterate by

$$
\Psi^{2}(x, u)=\Psi\left(x_{1}, u(h+\cdot)\right) .
$$

This procedure can be repeated iteratively, yielding a sequence of points $x_{0}, x_{1}, x_{2}, \ldots$ with the property

$$
x_{0}=x, \quad x_{j+1} \in \Psi\left(x_{j}, u(j h+\cdot)\right), j=0,1,2, \ldots
$$

As for the time discretization case we now define a concept of embedding. 
Definition 3.5. We say that the space discretization $\Psi$ is embedded into the perturbed system (2.1), (3.6) for some $\alpha>0$, if for any initial value $x \in \mathbb{R}^{d}$, any control function $u \in \mathcal{U}$ and any sequence of points $x_{j}$ satisfying (3.8) there exists an admissible perturbation function $w \in \mathcal{W}_{\alpha}$ such that

$$
\varphi(j h, x, u, j)=x_{j}
$$

holds for all $j \geq 0$.

Again we have to decide which class of perturbation functions we define to be admissible. Analogous to Lemma 3.1 we want to relate the perturbation range $\alpha$ to the discretization error, which here is given by the diameter $\operatorname{diam}(\mathcal{Q})$. It turns out that in order to realize this embedding the nonanticipative strategies $\mathcal{P}$ form a too restrictive class, which can be illustrated by Example 3.2: assume that the cell covering $\mathcal{Q}$ satisfies $\operatorname{diam}(\mathcal{Q})=2 \Delta$ and contains the cell $Q_{i^{*}}=[-\Delta, \Delta]$ centered around the origin. Then for any $x \in \mathbb{R}$ and any control $u \in \mathcal{U}$ there exists a sequence of points satisfying (3.8) and $\left|x_{j}\right| \geq \Delta$ for each $j \geq 1$, which is simply due to the fact that each cell $Q_{i}$ of $\mathcal{Q}$ contains a point $x$ with $|x| \geq \Delta$.

On the other hand, in Example 3.2 we have seen that if we choose $U=[-1,1]$ and $W=[-1 / 2,1 / 2]$ and nonanticipative perturbation strategies $w(t)=p[u](t)$ then for any $\tau>0$ we can find a control $u \in \mathcal{U}$ such that

$$
|\varphi(t, x, u, p[u])| \leq \tau / 2
$$

holds for each sufficiently large $t=j h>0$. Since $\left|x_{j}\right| \geq \Delta$ and $\tau>0$ is arbitrary, we can choose $\tau=\Delta / 4$ and it follows that for this class of perturbations (3.9) cannot be satisfied for $\alpha=1 / 2$, no matter how $\operatorname{small} \operatorname{diam}(\mathcal{Q})$ is.

The reason for this behavior lies in the fact that the inherent discrete time nature of the generation of the trajectory sequence (3.8) is not reflected in the strategy space $\mathcal{P}$. In order to overcome this problem it is necessary to relax the nonanticipation requirement in Definition 3.2 as follows.

Definition 3.6. For a time step $h>0$ we define the class of $h$-nonanticipative strategies by

$$
\mathcal{P}_{\alpha}^{h}:=\left\{\begin{array}{l|l}
p: \mathcal{U} \rightarrow \mathcal{W}_{\alpha} & \begin{array}{l}
\text { for all } t=j h, j \in \mathbb{Z} \text { the following implication holds: } \\
u_{1}(s)=u_{2}(s) \text { for almost all } s \in(-\infty, t] \Rightarrow \\
p\left[u_{1}\right](s)=p\left[u_{2}\right](s) \text { for almost all } s \in(-\infty, t]
\end{array}
\end{array}\right\}
$$

Hence, for any $j \in \mathbb{N}$ and any $s \in(j h,(j+1) h]$ the corresponding perturbation value $p[u](s)$ may be determined using all the values $u(t)$ for $t \in[0,(j+1) h]$ or, in other words, the perturbation function to be used on $(j h,(j+1) h]$ has to be fixed at time $(j+1) h$.

We return to Example 3.2 to illustrate this class of perturbations. 
Example 3.3. For the 1d system from the Examples 3.1 and 3.2, again with $U=$ $[-1,1]$ and $W=[-\alpha, \alpha]$ we investigate the effect of perturbations from $\mathcal{P}_{\alpha}^{h}$. We inductively define the perturbation strategies

$$
\left.p[u]\right|_{[j h,(j+1) h)} \equiv\left\{\begin{aligned}
-\alpha, & \text { if } \varphi(h, \varphi(j h, x, u, p[u]), u(j h+\cdot), 0) \leq 0 \\
\alpha, & \text { if } \varphi(h, \varphi(j h, x, u, p[u]), u(j h+\cdot), 0)>0 .
\end{aligned}\right.
$$

By induction over $j$ one sees that for any $u \in \mathcal{U}$ the resulting trajectory satisfies $|\varphi(j h, x, u, p[u])| \geq \alpha h$. This shows that in contrast to Example 3.2 the perturbations from $\mathcal{P}_{\alpha}^{h}$ are powerful enough to keep the system's trajectories persistently away from $A=\{0\}$, with the distance depending on both the size of $\alpha$ and on the time step $h$.

On the other hand, using the same $u$ as in Example 3.2 with $\tau=h$ yields a trajectory satisfying $|\varphi(j h, x, u, p[u])| \leq \alpha h$ for all sufficiently large $j$. Hence, in contrast to Example 3.1 under the perturbations from $\mathcal{P}_{\alpha}^{h}$ we are still able to control our system to a neighborhood of $A=\{0\}$ depending on $\alpha$ and $h$. Note that it is not possible to steer closer to $A$ by using $\tau=h / n$ for some $n \in \mathbb{N}$ in the construction of $u$ in Example 3.2, because this construction needs the values $\varphi(i \tau, x, u, p[u])$ for $i \in \mathbb{N}_{0}$, which are uncertain for $i \tau \in(j h,(j+1) j)$ because the values of the chosen strategy $p$ on the interval $(j h,(j+1) h]$ will only be fixed at time $(j+1) h$.

As expected, the controllability behavior of the system under these $h^{-}$ anticipative perturbations from $\mathcal{P}_{\alpha}^{h}$ lies between the behavior of Example 3.1 (full anticipation) and Example 3.2 (no anticipation). In particular, the effect of cells in a space discretization is reflected correctly as the following Lemma formally shows.

Lemma 3.2. Consider a trajectory (3.8) induced by a space discretization $\mathcal{Q}$ from Definition 3.3 with time step $h>0$. Then there exists a perturbation $p \in \mathcal{P}_{\alpha}^{h}$ with $\alpha=K \operatorname{diam}(\mathcal{Q}) / h$ for some $K>0$ such that the embedding property (3.9) holds for all $j \geq 0$ and all $u \in \mathcal{U}$.

Proof. For $t \in[0, h]$ we have no restriction in our choice of $p$, hence given $x_{0}, u$ and $x_{1}$ we can use

$$
\Delta x=\frac{x_{1}-\varphi\left(h, x_{0}, u, 0\right)}{h} \text { and } y(t)=\varphi\left(t, x_{0}, u, 0\right)+t \Delta x
$$

in order to define

$$
p[u](t)=f(\varphi(t, x, u, 0), u(t))-f(y(t), u(t))+\Delta x .
$$

A uniqueness argument similar to the proof of Lemma 3.1 shows that this perturbation yields the desired equality $\varphi\left(h, x_{0}, u, p[u]\right)=x_{1}$. A straightforward computation yields $\|p[u](t)\| \leq(1+L h)\|\Delta x\|$. Since $x_{1}$ and $\varphi(h, x, u, 0)$ lie in the same cell $Q_{i}$ with $\operatorname{diam}\left(Q_{i}\right) \leq \operatorname{diam}(\mathcal{Q})$ we obtain $\|\Delta x\| \leq \operatorname{diam}(\mathcal{Q}) / h$ which shows the assertion on the interval $[0, h]$. The extension to arbitrary intervals $[0, j h]$ now follows by induction over $j$. 
Remark 3.1. The embedding properties from Lemma 3.1 and 3.2 form one of the main motivations for the study of the robustness properties of the perturbed systems (2.1) or, more specifically, (3.6). The general structure of these systems allows for "universal" statements about robustness which can then be used for the analysis of large classes of numerical discretizations and algorithms. We will not pursue this analysis in detail here, instead we refer the interested reader to the Chapters 6 and 7 of Grüne ${ }^{6}$ as well as to Grüne ${ }^{8}$ and Kloeden and Kozyakin ${ }^{14}$.

\section{Robustness properties}

In this section we prove two robustness properties for asymptotic controllability under perturbations from $\mathcal{P}_{\alpha}^{h}$ from Definition 3.6. In fact, we will prove the results for a more general class of perturbations than $\mathcal{P}_{\alpha}^{h}$, namely

$$
\widetilde{\mathcal{P}}_{\alpha}^{h}:=\left\{\begin{array}{l|l}
p: \mathcal{U} \rightarrow \mathcal{W}_{\alpha} & \begin{array}{l}
\text { there exists a strictly increasing sequence }\left(t_{i}\right)_{i \in \mathbb{Z}} \text { with } \\
t_{i} \rightarrow \pm \infty \text { for } i \rightarrow \pm \infty \text { and } t_{i+1}-t_{i} \leq h \text { for all } i \in \mathbb{Z} \\
\text { such that for all } i \in \mathbb{Z} \text { the following implication holds: } \\
u_{1}(s)=u_{2}(s) \text { for almost all } s \in\left(-\infty, t_{i}\right] \Rightarrow \\
p\left[u_{1}\right](s)=p\left[u_{2}\right](s) \text { for almost all } s \in\left(-\infty, t_{i}\right]
\end{array}
\end{array}\right\}
$$

The sequence $\left(t_{i}\right)$ is called anticipation sequence of $p$. Note that this sequence need not be unique, e.g. for $p \in \mathcal{P}_{\alpha}$ any strictly increasing sequence $\left(t_{i}\right)_{i \in \mathbb{Z}}$ with the properties from above will be an anticipation sequence. Clearly, $\widetilde{\mathcal{P}}_{\alpha}^{h}$ contains $\mathcal{P}_{\alpha}^{h}$, thus robustness w.r.t. $\widetilde{\mathcal{P}}_{\alpha}^{h}$ implies robustness w.r.t. $\mathcal{P}_{\alpha}^{h}$. The main advantage of this larger set is that for any two perturbations strategy $p_{1}, p_{2} \in \widetilde{\mathcal{P}}_{\alpha}^{h}$ and any $\tau \in \mathbb{R}$ the strategy $p$ defined by the concatenation

$$
p[u](t)=\left\{\begin{array}{l}
p_{1}[u](t), t \in(-\infty, \tau) \\
p_{2}[u](t), t \in[\tau, \infty)
\end{array}\right.
$$

again lies in $\widetilde{\mathcal{P}}_{\alpha}^{h}$, i.e., this space is closed under concatenation for arbitrary times $\tau \in \mathbb{R}$. We will need this property in the proofs of the following results.

For our first result we need the following definition.

Definition 4.1. Consider $\alpha>0$. A compact set $A_{\alpha} \subset \mathbb{R}^{d}$ is called $\alpha$-controllable if

(i) for each $x \in A_{\alpha}$ and each $p \in \widetilde{\mathcal{P}}_{\alpha}^{h}$ there exists $u \in \mathcal{U}$ with $\varphi(t, x, u, p[u]) \in A_{\alpha}$ for all $t \geq 0$.

(ii) there exists a neighborhood $B_{\alpha}$ of $A_{\alpha}$ and a time $T_{\alpha}>0$ such that for each $x \in B_{\alpha}$ and each $p \in \widetilde{\mathcal{P}}_{\alpha}^{h}$ there exists a control function $u \in \mathcal{U}$ with $\varphi(t, x, u, p[u]) \in$ $A_{\alpha}$ for all $t \geq T_{\alpha}$.

Note that for $\alpha=0$ this definition is both stronger and weaker than the asymptotic controllability property in Definition 2.1: stronger, because it assumes controllability in finite time and weaker, because it does not impose a $\mathcal{K} \mathcal{L}$-estimate. In 
particular, it might happen that a point close to $A_{\alpha}$ might only be controllable to $A_{\alpha}$ by first steering far away from this set, which contradicts the existence of a $\mathcal{K} \mathcal{L}$ function $\beta$ as in Definition 2.1.

Definition 4.1 forms the basis for the following first robustness result.

Proposition 4.1. For every asymptotically controllable set $A$ with controllability neighborhood $B$ there exists $\gamma \in \mathcal{K}_{\infty}$ and $\alpha_{0}>0$ such that for each $\alpha \in\left[0, \alpha_{0}\right]$ there exists an $\alpha$-controllable set $A_{\alpha}$ with $B_{\alpha}=B$ and $H\left(A_{\alpha}, A\right) \leq \gamma(\alpha)$, where $H$ denotes the Hausdorff metric for compact subsets of $\mathbb{R}^{d}$.

In addition, for each $\alpha_{1}, \alpha_{2} \in\left[0, \alpha_{0}\right]$ with $\alpha_{2}>\alpha_{1}$, any $x \in A_{\alpha_{2}}$ and any $p \in \widetilde{\mathcal{P}}_{\alpha_{1}}^{h}$ the control $u$ steering $x$ to $A_{\alpha_{1}}$ can be chosen such that

$$
\varphi(t, x, u, p[u]) \in A_{\alpha_{2}}
$$

holds for all $t \geq 0$.

Furthermore, there exists a function $\chi \in \mathcal{K}_{\infty}$ such that for each $\alpha>0$ and each two points $x \in A$ and $y \notin A_{\alpha}$ the inequality $\|x-y\| \geq \chi(\alpha)$ holds.

Proof. For each $x \in B$ we denote by $u_{x} \in \mathcal{U}$ the control satisfying

$$
\left\|\varphi\left(t, x, u_{x}, 0\right)\right\|_{A} \leq \beta\left(\|x\|_{A}, t\right)
$$

for all $t \geq 0$. We set $r_{0}=H(B, A)$. For all $r \in\left(0, r_{0}\right]$ we define

$$
T(r)=\min \left\{t \geq 0 \mid \beta(s, t) \leq \frac{s}{4} \text { for all } s \in\left[r, r_{0}\right]\right\} .
$$

Then $T(r)$ is finite for all $r \in(0, r]$ and monotone decreasing. The monotonicity of $\beta$ implies

$$
\beta(s, T(r)+t) \leq \frac{r}{4}
$$

for all $t \geq 0$, all $r \in\left(0, r_{0}\right]$ and all $s \in[0, r]$. Via

$$
\widetilde{T}(r)=\sup _{s>0}\{T(s)-|s-r|\}+h
$$

we obtain a function $\widetilde{T}(r) \geq T(r)+h$, which is also monotone decreasing but in addition continuous, even globally Lipschitz with constant $L=1$.

We set $\alpha_{0}$ to

$$
\alpha_{0}:=\frac{r_{0}}{4 \widetilde{T}\left(r_{0}\right) e^{\widetilde{T}\left(r_{0}\right)}}
$$

and define a function $r(\alpha)$ for $\alpha \in\left(0, \alpha_{0}\right]$ by setting $r(\alpha)=r>0$ for $r$ being the (unique) solution of the equation

$$
\widetilde{T}(r) e^{\widetilde{T}(r)} \alpha=\frac{r}{4} .
$$

Observe that the monotonicity and continuity properties of the functions involved in (4.10) imply continuity of $r(\alpha)$, furthermore, this function is strictly increasing in $\alpha$ with $r(\alpha) \rightarrow 0$ for $\alpha \rightarrow 0$. We set $r(0)=0$. For any perturbation $p$ we denote 
by $T_{p}(r)$ the smallest time $t_{i} \geq T(r)$ in the anticipation sequence of $p$. Note that $T_{p}(r) \leq \widetilde{T}(r)$ holds.

From the Gronwall Lemma we obtain for each $x \in B$ and $p \in \widetilde{\mathcal{P}}_{\alpha}^{h}$ the inequality

$$
\begin{aligned}
\left\|\varphi\left(t, x, u_{x}, p\left[u_{x}\right]\right)\right\|_{A} & \leq\left\|\varphi\left(t, x, u_{x}, p\left[u_{x}\right]\right)-\varphi\left(t, x, u_{x}, 0\right)\right\|+\left\|\varphi\left(t, x, u_{x}, 0\right)\right\|_{A} \\
& \leq \alpha t e^{L t}+\beta\left(\|x\|_{A}, t\right) .
\end{aligned}
$$

For $t \leq \widetilde{T}(r(\alpha))$ we have

$$
\alpha t e^{L t} \leq \alpha \widetilde{T}(r(\alpha)) e^{L \widetilde{T}(r(\alpha))}=\frac{r(\alpha)}{4} .
$$

Now we consider the sets

$$
D_{\alpha}=\overline{\mathcal{B}(r(\alpha), A)} .
$$

For $x \in D_{\alpha}$ we have $d(x, A) \leq r(\alpha)$ and thus by Gronwall's Lemma, (4.11) and the definition of $\widetilde{T}(r)$ the estimate

$$
\left\|\varphi\left(T_{p}(r(\alpha)), x, u_{x}, p\left[u_{x}\right]\right)\right\|_{A} \leq \frac{r(\alpha)}{4}+\underbrace{\beta\left(\|x\|_{A}, T(r(\alpha))\right)}_{\leq r(\alpha) / 4} \leq \frac{r(\alpha)}{2}<r(\alpha),
$$

thus

$$
\varphi\left(T_{p}(r(\alpha)), x, u_{x}, p\left[u_{x}\right]\right) \in D_{\alpha} .
$$

For the times $t$ with $0 \leq t \leq T_{p}(r(\alpha))$ we have

$$
\varphi\left(t, x, u_{x}, p\left[u_{x}\right]\right) \|_{A} \leq \frac{r(\alpha)}{4}+\beta\left(\|x\|_{A}, t\right) \leq \frac{r(\alpha)}{4}+\beta(r(\alpha), 0) .
$$

Denoting $x_{1}:=\varphi\left(T_{p}(r(\alpha)), x, u_{x}, p\left[u_{x}\right]\right)$ by (4.12) we can continue

$$
\left\|\varphi\left(t, x_{1}, u_{x_{1}}, p\left[u_{x_{1}}\right]\right)\right\|_{A} \leq \frac{r(\alpha)}{4}+\beta\left(\left\|x_{1}\right\|_{A}, t\right) \leq \frac{r(\alpha)}{4}+\beta(r(\alpha), 0) .
$$

Thus, proceeding inductively (note that this is possible due to the fact that $T_{p}(r(\alpha)$ ) is from the anticipation sequence of $p$ ), we can construct $u$ such that (4.13) holds for all $t \geq 0$. Defining

$$
A_{\alpha}=\bigcup_{t \geq 0} \bigcup_{x \in D_{\alpha}, p \in \widetilde{\mathcal{P}}_{\alpha}^{h}}\{\varphi(t, x, u, p[u])\}
$$

we obtain a set such that for each point $x \in A_{\alpha}$ and each $p \in \widetilde{\mathcal{P}}_{\alpha}^{h}$ there exists $u \in \mathcal{U}$ with

$$
\varphi\left((t, x, u, p[u]) \in A_{\alpha}\right.
$$

for all $t \geq 0$. Furthermore, we obtain the inequality

$$
H\left(A_{\alpha}, A\right) \leq \frac{r(\alpha)}{4}+\beta(r(\alpha), 0)=: \gamma(\alpha)
$$

Observe that this $\gamma$ only depends on $\beta$ and $r(\alpha)$, and thus — via Gronwall's Lemma - on $\beta, r_{0}=H(B, A)$ and the Lipschitz constant $L$ of $f$. 
The $\alpha$-controllability property to $A_{\alpha}$ is shown analogously with the same inductively defined control functions $u$, again using Gronwall's Lemma to show that each point $x \in B$ can be controlled to $D_{\alpha} \subseteq A_{\alpha}$ in finite time.

The fact that each point from $A_{\alpha_{2}}$ can be controlled to $\alpha_{1}$ without leaving $A_{\alpha_{2}}$ follows from the construction of the control functions $u$, which are the same in both parts of the proof.

Finally, the existence of the function $\chi$ follows from the fact that each $A_{\alpha}$ contains the closed ball $D_{\alpha}=\overline{\mathcal{B}(r(\alpha), A)}$, i.e., we can choose $\chi(\alpha)=r(\alpha)$.

Remark 4.1. For arbitrarily perturbed systems (2.1) is it in general not possible to derive a perturbed analogue of Definition 2.1 for the sets $A_{\alpha}$ from Proposition 4.1 , i.e., an estimate of the type

$$
\|\varphi(t, x, u, p[u])\|_{A_{\alpha}} \leq \tilde{\beta}\left(\|x\|_{A_{\alpha}}, t\right),
$$

for some $\tilde{\beta} \in \mathcal{K} \mathcal{L}$ because it might be necessary to steer far away from $A_{\alpha}$ before we can actually control the system to $A_{\alpha}$. For inflated systems (3.6), however, one can exploit the particular perturbation structure in order to conclude (4.14) for all $p \in \widetilde{\mathcal{P}}_{\alpha^{\prime}}^{h}$ with $\alpha^{\prime} \in(0, \alpha)$ arbitrary and $\tilde{\beta}$ depending on $\alpha^{\prime}$, see Proposition 4.7.4 in Grüne $^{6}$.

The major drawback of Proposition 4.1 in our setting is that the time-varying nature of the perturbations $w(t)=p[u](t)$ is not reflected: the statement only takes into account the maximal norm of $p$ but not the change of $p$ in time. The following definition is motivated by the notion of input-to-state dynamical stability (ISDS) ${ }^{7}$ which in turn is based on the input-to-state stability property (ISS) introduced by Sontag ${ }^{17,19}$. For its formulation we need so called $\mathcal{K} \mathcal{L D}$ functions, which are functions $\mu \in \mathcal{K} \mathcal{L}$ which in addition satisfy $\mu(r, 0)=r$ and $\mu(r, t+s)=\mu(\mu(r, t), s)$ for all $r, t, s \geq 0$, i.e., they are semi dynamical systems.

Definition 4.2. An asymptotically controllable set $A$ is called controlled input-tostate dynamically stable (cISDS) if there exist $\alpha_{0}>0, \gamma, \sigma \in \mathcal{K}_{\infty}, \mu \in \mathcal{K} \mathcal{L D}$ and a neighborhood $B$ of $A$, such that for each $x \in B$ and each perturbation strategy $p \in \widetilde{\mathcal{P}}_{\alpha_{0}}^{h}$ with anticipation sequence $\left(t_{i}\right)$ there exists $u \in \mathcal{U}$ with

$$
\|\varphi(t, x, u, p[u])\|_{A} \leq \max \left\{\mu\left(\sigma\left(\|x\|_{A}\right), t\right), \nu(p, t)\right\}
$$

for all $t=t_{i} \geq 0$ from the sequence $\left(t_{i}\right)$, where

$$
\nu(p, t)=\sup _{u \in \mathcal{U}} \operatorname{ess}_{\tau \in[0, t]} \sup \mu(\gamma(\|p[u](\tau)\|), t-\tau)
$$

Our main result states that each asymptotically controllable set has the cISDS property.

Theorem 4.1. Every asymptotically controllable set $A$ is cISDS for suitable $\alpha_{0}>$ $0, \gamma, \sigma \in \mathcal{K}_{\infty}$ and $\mu \in \mathcal{K} \mathcal{L} \mathcal{D}$. 
Proof. We show the assertion by constructing a function $V: B \rightarrow \mathbb{R}$ with the property

$$
\|x\|_{A} \leq V(x) \leq \sigma\left(\|x\|_{A}\right)
$$

and

$$
V\left(\varphi(t, x, u, p[u]) \|_{A} \leq \max \{\mu(V(x), t), \nu(p, t)\}\right.
$$

for any $x \in O, p \in \widetilde{\mathcal{P}}_{\alpha}^{h}, t=t_{i} \geq 0, \nu$ from Definition 4.2 and a suitable $u \in \mathcal{U}$ depending on $x, p$ and the sequence $\left(t_{i}\right)$. From these inequalities the asserted cISDS property follows immediately.

Without loss of generality we can assume that $B$ is controlled invariant, i.e., that for each $x \in B$ and each $p \in \widetilde{\mathcal{P}}_{\alpha_{0}}^{h}$ there exists $u \in \mathcal{U}$ with $\varphi(t, x, u, p[u]) \in B$ for all $t \geq 0$.

In order to construct $V$ we use Proposition 4.1. We denote the $\mathcal{K}_{\infty}$ function from Proposition 4.1 by $\bar{\gamma}$ and consider an arbitrary $\tilde{\gamma} \in \mathcal{K}_{\infty}$ with $\tilde{\gamma}>\bar{\gamma}$ for $r>0$. We define $\eta=\bar{\gamma}^{-1} \circ \tilde{\gamma} \in \mathcal{K}_{\infty}$ and inductively define a two sided sequence $b_{i}, i \in \mathbb{Z}$, by setting $b_{0}=1$ and

$$
b_{i+1}=\eta\left(b_{i}\right) \text { and } b_{i-1}=\eta^{-1}\left(b_{i}\right)
$$

for $i \in \mathbb{Z}$. One easily computes that

$$
b_{i}<b_{i+1} \text { and } \tilde{\gamma}\left(b_{i}\right)=\bar{\gamma}\left(b_{i+1}\right)
$$

as well as $b_{i} \rightarrow 0$ for $i \rightarrow-\infty$ and $b_{i} \rightarrow \infty$ for $i \rightarrow \infty$ holds. We abbreviate $r_{i}=\bar{\gamma}\left(b_{i}\right)$ and $\tilde{r}_{i}=\tilde{\gamma}\left(b_{i}\right)$ and set $a_{i}=\max \left\{b_{i}, \alpha_{0}\right\}$.

Now we define the sets

$$
B_{i}:=A_{a_{i}}
$$

for $A_{\alpha}$ from Proposition 4.1. These sets satisfy $H\left(B_{i}, A\right) \leq r_{i}$ and - by their construction in the proof of Proposition $4.1-B_{i} \subseteq B_{i+1}$. Furthermore, for each $x \in B_{i}$ and each $p \in \widetilde{\mathcal{P}}_{a_{i-1}}^{h}$ there exists $u_{x, p} \in \mathcal{U}$ with

$$
\varphi\left(t, x, u_{x, p}, p\left[u_{x, p}\right]\right) \in B_{i}
$$

for all $t \geq 0$ and

$$
\varphi\left(t, x, u_{x, p}, p\left[u_{x, p}\right]\right) \in B_{i-1}
$$

for all $t \geq T_{a_{i-1}}$ from Definition 4.1(ii).

We set $\Delta t_{i}=T_{a_{i-1}}+h$ and define $V$ as follows: for each $r \in\left[\tilde{r}_{i-1}, \tilde{r}_{i}\right]$ we define the sets

$$
C_{r}:=\bigcup_{p \in \tilde{\mathcal{P}}_{a_{i-1}}^{h}, x \in B_{i}} \varphi\left(\frac{\tilde{r}_{i}-r}{\tilde{r}_{i}-\tilde{r}_{i-1}} \Delta t_{i}, x, u_{x, p}, p\left[u_{x, p}\right]\right) \cup B_{i-1} .
$$

Observe that the choice of $\Delta t_{i}$ implies

$$
C_{r}=B_{i-1} \quad \text { for } \quad r \leq \tilde{r}_{i}-\frac{\Delta t_{i}-h}{\Delta t_{i}}\left(\tilde{r}_{i}-\tilde{r}_{i-1}\right) .
$$


Now we obtain our function $V$ by setting

$$
V(x):=\inf \left\{r>0 \mid x \in C_{r}\right\} .
$$

We first show that (4.15) holds. From Proposition 4.1 we know that each $x \in B_{i}$ satisfies $\|x\|_{A} \leq \bar{\gamma}\left(b_{i}\right)=\tilde{\gamma}\left(b_{i-1}\right)$. For $r \in\left[\tilde{r}_{i-1}, \tilde{r}_{i}\right]$ and $x \in C_{r}$ we obtain $x \in B_{i}$ and hence $\|x\|_{A} \leq \tilde{\gamma}\left(b_{i-1}\right)=\tilde{r}_{i-1} \leq r$. This yields the implication $d(x, A)=r \Rightarrow$ $x \notin C_{\tilde{r}}$ for all $\tilde{r}<r \Rightarrow V(x) \geq \tilde{r}$ for all $\tilde{r}<r \Rightarrow V(x) \geq r$ and consequently

$$
V(x) \geq d(x, A) \text {. }
$$

For the upper bound recall that Proposition 4.1 yields $x \notin B_{i} \Rightarrow\|x\|_{A} \geq \chi\left(b_{i}\right)$. For $r \in\left[\tilde{r}_{i-1}, \tilde{r}_{i}\right]$ and $x \notin C_{r}$ we obtain $x \notin B_{i-1}$ and thus

$$
\|x\|_{A}>\chi\left(b_{i-1}\right) \geq \chi \circ \tilde{\gamma}^{-1} \circ \bar{\gamma} \circ \tilde{\gamma}^{-1}\left(\tilde{r}_{i}\right) \geq \chi \circ \tilde{\gamma}^{-1} \circ \bar{\gamma} \circ \tilde{\gamma}^{-1}(r) .
$$

Defining $\sigma(r):=\tilde{\gamma} \circ \bar{\gamma}^{-1} \circ \tilde{\gamma} \circ \chi^{-1}$ we obtain

$$
V(x)>r \Rightarrow x \notin C_{r} \Rightarrow \sigma\left(\|x\|_{A}\right)>r
$$

and finally

$$
V(x) \leq \sigma\left(\|x\|_{A}\right) .
$$

It remains to show (4.16), in particular we have to define $\mu$. To this end for $r=\tilde{r}_{i}$ and $t \in\left[0, \Delta t_{i}\right]$ we set

$$
\mu(r, t)=r-t \frac{\tilde{r}_{i}-\tilde{r}_{i-1}}{\Delta t_{i}}
$$

and extend this map for all $t, r \geq 0$ such that the dynamical systems property $\mu(r, t+s)=\mu(\mu(r, t), s)$ holds. This construction yields

$$
\frac{\tilde{r}_{i}-\mu(r, t)}{\tilde{r}_{i}-\tilde{r}_{i-1}} \Delta t_{i} \leq \frac{\tilde{r}_{i}-\left(r-t \frac{\tilde{r}_{i}-\tilde{r}_{i-1}}{\Delta t_{i}}\right)}{\tilde{r}_{i}-\tilde{r}_{i-1}} \Delta t_{i}=\frac{\tilde{r}_{i}-r}{\tilde{r}_{i}-\tilde{r}_{i-1}} \Delta t_{i}+t .
$$

For any $r \in\left(\tilde{r}_{i-1}, \tilde{r}_{i}\right]$ we define $t(r):=\frac{\tilde{r}_{i}-r}{\tilde{r}_{i}-\tilde{r}_{i-1}} \Delta t_{i}$ which implies

$$
\mu\left(\tilde{r}_{i}, t(r)\right)=r .
$$

From the construction of $C_{r}$ each $x^{*} \in C_{r}$ is either in $B_{i-1}$ or it is the image of $\varphi$ for some point $x \in B_{i}$, some perturbation $p$ and control $u_{x, p}$ at time $t(r)$. Now consider a perturbation $p^{*} \in \widetilde{\mathcal{P}}_{a_{i-1}}^{h}$ and a time $t \in\left[0, \Delta t_{i}-t(r)\right]$. In the first case we choose $u^{*}=u_{x^{*}, p^{*}}$ which keeps the resulting trajectory inside $B_{i-1}$. In the second case, by the concatenation property of $\widetilde{\mathcal{P}}_{a_{i-1}}^{h}$ for all $x \in C_{r}$ and all $t \in\left[0, \Delta t_{i}-t(r)\right]$ we find a perturbation $p \in \widetilde{\mathcal{P}}_{a_{i-1}}^{h}$ which coincides with $p^{*}$ on $[t(r), \infty)$ and we choose $u^{*}=u_{p, x}(t(r)+\cdot)$. This yields

$$
\begin{aligned}
\varphi\left(t, x^{*}, u^{*}, p\left[u^{*}\right]\right) & \in \bigcup_{p \in \tilde{\mathcal{P}}_{a_{i-1}}^{h}, x \in B_{i}} \varphi\left(t(r)+t, x, u_{x, p}, p\left[u_{x, p}\right]\right) \cup B_{i-1} \\
& \subseteq \bigcup_{p \in \tilde{\mathcal{P}}_{a_{i-1}}^{h}, x \in B_{i}} \varphi\left(\frac{\tilde{r}_{i}-\mu(r, t)}{\tilde{r}_{i}-\tilde{r}_{i-1}} \Delta t_{i}, x, u_{x, p}, p\left[u_{x, p}\right]\right) \cup B_{i-1} \\
& \subseteq C_{\mu(r, t)}
\end{aligned}
$$


for all $t \in\left[0, \Delta t_{i}-t(r)\right]$.

Finally, we use (4.18) to show that (4.16) holds for the robustness gain $\gamma \in \mathcal{K}$ given by $\gamma=\eta \circ \tilde{\gamma}$. Note that this definition implies $\gamma \circ \tilde{\gamma}^{-1}\left(\tilde{r}_{i-1}\right)=\tilde{r}_{i}$. We consider $x \in B$, some perturbation $p \in \widetilde{\mathcal{P}}_{\alpha_{0}}^{h}$ and the smallest positive time $t_{j}>0$ from the anticipation sequence $\left(t_{i}\right)$ of $p$. We pick the minimal value $r \geq 0$ with the following properties:

$$
V(x) \leq r, \quad q_{t}:=\sup _{u \in \mathcal{U}} \operatorname{ess} \sup \tilde{\tau \in\left[0, t_{j}\right]} \tilde{\gamma}(\|p[u](\tau)\|) \leq r
$$

and

$$
r \in\left[\tilde{r}_{i}-\frac{\Delta t_{i}-h}{\Delta t_{i}}\left(\tilde{r}_{i}-\tilde{r}_{i-1}\right), \tilde{r}_{i}\right]
$$

for some $i \in \mathbb{Z}$. The minimality of $r$ implies that either $V(x)=r$ or $q_{t}=r$ or $r=\tilde{r}_{i}-\frac{\Delta t_{i}-h}{\Delta t_{i}}\left(\tilde{r}_{i}-\tilde{r}_{i-1}\right)$ must hold. Since (4.17) implies that $V$ cannot assume values in the intervals given in (4.19), we obtain that if $V(x)<r$ and $q_{t}<r$ hold then $q_{t}>\tilde{r}_{i-1}$ must hold, because otherwise the minimal $r$ would satisfy $r \leq \tilde{r}_{i-1}$. From $\gamma \circ \tilde{\gamma}^{-1}\left(\tilde{r}_{i-1}\right)=\tilde{r}_{i}$ and the definition of $\nu$ we obtain $\nu\left(p, t_{j}\right) \geq \mu\left(\tilde{r}_{i}, t_{j}\right) \geq \mu\left(r, t_{j}\right)$ because $\mu$ is monotone increasing in its first argument, and consequently

$$
\mu\left(r, t_{j}\right) \leq \max \left\{\mu\left(V(x), t_{j}\right), \nu\left(p, t_{j}\right)\right\} .
$$

Since $r$ satisfies 4.19 and $V$ only assumes values in the intervals from (4.19) we can conclude that we either have $r=\tilde{r}_{i}$ implying $x \in C_{r}$, or $x \in C_{\tilde{r}}$ for all $\tilde{r} \geq r$. In any case we can pick $\tilde{r}$ from the interval in (4.19) with $x \in C_{\tilde{r}}$ for which the definition of $t(r)$ implies $\Delta t_{i}-t(\tilde{r}) \geq h$, i.e., in particular $t_{j} \in\left[0, \Delta t_{i}-t(\tilde{r})\right]$. Hence we can use $u=u^{*} \in \mathcal{U}$ from (4.18) with $x^{*}=x$ in order to obtain

$$
\varphi\left(t_{j}, x, u, p[u]\right) \in C_{\mu\left(\tilde{r}, t_{j}\right)} .
$$

For $V$ this implies

$$
V\left(\varphi\left(t_{j}, x, u, p[u]\right)\right) \leq \mu\left(\tilde{r}, t_{j}\right),
$$

and since either $r=\tilde{r}$ holds or $r>\tilde{r}$ is arbitrary we obtain

$$
V(\varphi(t, x, u, p[u])) \leq \mu(r, t) .
$$

Together with (4.20) this proves (4.16) for $t=t_{j}$.

In order to continue this estimate for larger $t_{i}$ from the anticipation sequence $\left(t_{i}\right)$ we consider the next anticipation time $t_{j+1}$ and repeat the construction on $\left[t_{j}, t_{j+1}\right]$ with $x=\varphi\left(t_{j}, x, u, p[u]\right)$ and $p[u]=p[u]\left(t_{j}+\cdot\right)$ to conclude the inequality (4.16) for all $t \in\left[0, t_{j+1}\right]$. Proceeding inductively we obtain (4.16) for all anticipation times $t_{i} \geq 0$.

Remark 4.2. The cISDS property contains many interesting features of the system in one compact formula. For instance, one immediately obtains that if $\sup _{u \in \mathcal{U}} p[u](t) \rightarrow 0$ for $t \rightarrow \infty$ then the system can be asymptotically controlled to 
$A$. In the context of discretizations this is an important feature to analyze schemes with time-varying discretization errors like adaptive subdivision schemes as developed by Dellnitz and Hohmann ${ }^{3}$ and Szolnoki ${ }^{21}$, see also Chapter 6 of Grüne ${ }^{6}$. The cISDS property also contains Proposition 4.1 because one can easily identify the sets $A_{\alpha}$ as sublevel sets of $V$. In fact, the existence of the function $V$, which acts as a cISDS Lyapunov function, is the main motivation for the specific form of the cISDS formulation, cf. Grüne ${ }^{7}$ for details on this in the uncontrolled case. Note that the function $V$ constructed above is in general discontinuous and it is an open question whether and under which conditions one can find a continuous or even differentiable cISDS Lyapunov function $V$.

Remark 4.3. Note that for $p \in \mathcal{P}_{\alpha}$ we can choose an arbitrary anticipation sequence $\left(t_{i}\right)$ for which the cISDS estimate holds, such that we recover the wISDS definition from Chapter 4 of Grüne ${ }^{6}$. Note that for the times $t \in\left(t_{i}, t_{i+1}\right)$ in between the anticipation times the proof above yields the estimate

$$
\|\varphi(t, x, u, p[u])\|_{A} \leq \max \left\{\mu\left(\sigma\left(\|x\|_{A}\right), t\right), \nu\left(p, t_{i+1}\right)\right\},
$$

i.e., we obtain a similar estimate involving "future" perturbation values from the interval $\left(t_{i}, t_{i+1}\right)$.

Acknowledgment: I would like to thank Tony Michel for information about the history of $\mathcal{K}$ and $\mathcal{K} \mathcal{L}$ functions.

\section{References}

1. M. Bardi and I. Capuzzo Dolcetta. Optimal Control and Viscosity Solutions of Hamilton-Jacobi-Bellman equations. Birkhäuser, Boston, 1997.

2. F. Colonius and W. Kliemann. The Dynamics of Control. Birkhäuser, Boston, 2000.

3. M. Dellnitz and A. Hohmann. A subdivision algorithm for the computation of unstable manifolds and global attractors. Numer. Math., 75:293-317, 1997.

4. M. Dellnitz and O. Junge. Set oriented numerical methods for dynamical systems. In B. Fiedler, editor, Handbook of Dynamical Systems. Volume 2, pages 221-264. Elsevier, Amsterdam, 2002.

5. R. Ferretti. High-order approximations of linear control systems via Runge-Kutta schemes. Computing, 58(4):351-364, 1997.

6. L. Grüne. Asymptotic Behavior of Dynamical and Control Systems under Perturbation and Discretization. Lecture Notes in Mathematics, Vol. 1783. Springer-Verlag, 2002.

7. L. Grüne. Input-to-state dynamical stability and its Lyapunov function characterization. IEEE Trans. Autom. Control, 47:1499-1504, 2002.

8. L. Grüne. Attraction rates, robustness and discretization of attractors. SIAM J. Numer. Anal., 41:2096-2113, 2003.

9. L. Grüne and P. E. Kloeden. Higher order numerical schemes for affinely controlled nonlinear systems. Numer. Math., 89:669-690, 2001.

10. W. Hahn. Theorie und Anwendung der direkten Methode von Ljapunov. Ergebnisse der Mathematik und ihrer Grenzgebiete 22. Springer-Verlag Berlin, Göttingen, Heidelberg, 1959.

11. W. Hahn. Stability of Motion. Springer-Verlag Berlin, Heidelberg, 1967. 
12. E. Kamke. Zur Theorie der Systeme gewöhnlicher Differentialgleichungen. Acta Math., 58:57-85, 1932.

13. P. E. Kloeden. Eventual stability in general control systems. J. Differ. Equations, 19:106-124, 1975.

14. P. E. Kloeden and V. S. Kozyakin. The inflation of attractors and their discretization: the autonomous case. Nonlinear Anal., Theory Methods Appl., 40:333-343, 2000.

15. P. E. Kloeden and J. Lorenz. Stable attracting sets in dynamical systems and their one-step discretizations. SIAM J. Numer. Anal., 23:986-995, 1986.

16. M. Müller. Über das Fundamentaltheorem in der Theorie der gewöhnlichen Differentialgleichungen. Math. Z., 26:619-645, 1926.

17. E. D. Sontag. Smooth stabilization implies coprime factorization. IEEE Trans. Autom. Control, 34:435-443, 1989.

18. E. D. Sontag. Stability and stabilization: Discontinuities and the effect of disturbances. In Proc. NATO Advanced Study Institute "Nonlinear Analysis, Differential Equations, and Control" (Montreal, Jul/Aug 1998). Kluwer, 1999.

19. E. D. Sontag. The ISS philosophy as a unifying framework for stability-like behavior. In A. Isidori, F. Lamnabhi-Lagarrigue, and W. Respondek, editors, Nonlinear Control in the Year 2000, Volume 2, Lecture Notes in Control and Information Sciences 259, pages 443-468. NCN, Springer Verlag, London, 2000.

20. A. M. Stuart and A. R. Humphries. Dynamical Systems and Numerical Analysis. Cambridge University Press, 1996.

21. D. Szolnoki. Set oriented methods for computing reachable sets and control sets. Discrete Contin. Dyn. Syst. Ser. B, 3(3):361-382, 2003.

22. S. Szufla. On the equation $x^{\prime}=f(t, x)$ in locally convex spaces. Math. Nachr., 118:179185, 1984.

23. P. P. Varaiya. On the existence of solutions to a differential game. SIAM J. Control, 5:153-162, 1967. 